\title{
Virtual Reality in Cognitive and Perceptual Rehabilitation - A Promising Approach
}

\author{
Ruby Aikat ${ }^{1 \#^{*}}$, Vandana Dua ${ }^{2 \#}$ and Nishu Tyagi ${ }^{3 \#}$ \\ ${ }^{1}$ Indian Spinal Injuries Centre, Institute of Rehabilitation Sciences, New Delhi, India \\ ${ }^{2}$ Neurosciences CNC Centre, All India Institute of Medical Sciences, Ansari Nagar, New Delhi, India \\ ${ }^{3}$ Telehealth Unit (E-Seva Centre), Indian Spinal Injuries Centre, New Delhi, India
}

"Corresponding author: Ruby Aikat, Assistant Professor, Indian Spinal Injuries Centre Institute of Rehabilitation Sciences, New Delhi, India, E-mail: ruby_aikat@rediffmail.com

\# All Authors Contributed equally

Rec date: Jul 22, 2016; Acc date: Jul 29, 2016; Pub date: Jul 30, 2016

Copyright: (c) 2016 Aikat $\mathrm{R}$, et al. This is an open-access article distributed under the terms of the Creative Commons Attribution License, which permits unrestricted use, distribution, and reproduction in any medium, provided the original author and source are credited.

\section{Opinion}

In modern medical era, the paradigm of rehabilitation bench upon functional outcomes. The conventional approaches primarily dealing with physical issues in terms of sensory-motor deficits of patients with neurological disorders seem insufficient. Cognitive and perceptual rehabilitation is the one which enables the individual to utilize various sensory cues from environment together with its understanding, judgement and memory for achieving meaningful gains. It is important that the rehabilitation approaches do not underestimate the importance of this area of rehabilitation because cognitive and perceptual skills are, in a way, considered prerequisite skills to even physical performances.

Virtual reality (VR) is one, amongst other modalities, with immense potential to be used in comprehensive neurological rehabilitation, though most of its utilization has been done in terms of physical training. Its concept and techniques has been used in the field of rehabilitation since years. However its potential in terms of cognitive and perceptual rehabilitation has been under-explored. VR typically refers to the use of interactive simulations created with computer hardware and software to present users with opportunities to engage in environments that appear to be and feel similar to real-world objects and events [1]. VR follows the theories of mental imagery not only for physical gains but also for cognitive and perceptual improvement. Our recent review on mental imagery focused on the use of mental imagery in physical gains [2]. Virtual reality, unlike that, follows the theories of mental imagery not only for physical gains but also for cognitive and perceptual improvement. It is based on the concept of neuro-plasticity which states that structural and functional reorganization follows any damage to central nervous system. Various researches have shown that this re-organization can be enhanced by exposing the individual to stimulation situations with the use of virtual reality.

The technique of virtual reality training could be immersive or nonimmersive type. Immersive type means the client is basically immersed in a virtual environment. The advantage of immersive systems is the user's sense of being a part of the virtual world. In non-immersive systems, the VR system consists of a display device, mouse, keyboard and possibly joysticks, tactile and force sensors. The multi-sensory illusion is created via visual, tactile and auditory feedback. Visual feedback is traditionally given by computer screens, large screen projection, wall projectors and head-mounted displays. Acoustic feedback in mono or stereo sound can be provided by speakers, headphones or other sophisticated surround sound systems. Tactile feedback provided by robotic actuators or haptic (tactile) gloves that vibrate against the skin or within the device [3]. Conventionally the 2 dimensional (2D) methods of virtual reality/ gaming systems are being used. With the advent of newer technologies, the 3D methods are now being developed as a part of the virtual environment training system. A computer-generated 3D object should be a) an accurate description of the model being designed $b$ ) presented in a realistic and integrated format, so that it can be visualized and interpreted without introducing uncertainty regarding the represented properties [4]. To ensure that the displayed 3D image will satisfy these requirements, it is important to focus on which perceptual/cognitive operation is performed by the user during the design process.

Virtual reality has gained broader recognition in western countries for the evaluation and treatment of many parameters like sitting and standing balance, hand functions, shoulder functions, coordination, fitness and endurance training etc. in population of different diagnosis like stroke, total knee replacement, hip replacement, ligament injuries, spinal cord injury, elderly and so on. In developing country like India, the use of virtual reality is in a nascent stage. Professionals have begun to make use of virtual reality in multiple diagnoses with promising results. In our clinical practice, we are providing VR based therapy using Nintendo Wiifit(which is a 2D method of virtual reality training) for conditions like spinal cord injury, elderly, total knee replacement, and anterior cruciate ligament tear, children with cerebral palsy to address issues of sitting balance, standing balance, and shoulder - hand functions specifically; and we have seen wonderful results. We have recently also worked on a group of clients with stroke and Parkinson's disease and found positive results in dynamic standing balance [5]. Interestingly, caregivers and patients are purchasing VR tools and CDs for their individual rehabilitation goals to be carried out as a part of their home therapy programs.

Also, recently we have begun to explore the VR software specifically designed to address the cognitive- perceptual issues for clients with stroke/head injury and in a couple of children with developmental delay. We have observed a drastic difference in the active participation levels of the clients in the therapy sessions. As opposed to the conventional methods of cognitive-perceptual rehabilitation, where there has always been the risk of lack of motivation, reduced arousal levels, diminished threshold of visual and auditory stimuli from the environment leading to reduced participation in therapy sessions, inability to sustain attention in the tasks, to name a few; a virtual environment training session not only captures the attention of the client and sustains it for much longer duration; it also keeps the motivation level maintained. Since the client gets constant auditory 
Page 2 of 2

and visual feedback of his performances immediately, he is motivated to move on to the next task. Another added advantage of such software is that the difficulty level of each cognitive task can be graded, rendering this method of virtual reality training suitable for all age groups, and clients with different educational and cultural backgrounds. With VR, patients feel safe and get an opportunity to practice their given task in a safe and simulated environment. The therapists have the means to provide a variety of environments similar to those encountered in real life situations which may help the patients to easily adapt to their real life situations. Through this system of VR, patients not only get the feedback of their performance but it also encourages them to get involved in the self-reward process in which they can beat their own scores in subsequent attempts, which, in turn, leads to the activation of reward circuitry process and motivation for further accomplishment of planned goals [6]. Its application is expanding its roots in cognitive and perceptual rehabilitation for the patients who get agitated with monotonous conventional therapy sessions with less feedback and motivational factors.

Nowadays, developers are targeting the use of virtual reality in the patients' home as well under the close supervision of professionals. Now the question arises about the feasibility and practical applicability of the same. Is this possible? If yes, then how. For this, we, at our centre, are using tele-rehabilitation technologies, which are being commonly used in the developed nations. Virtual reality games can be easily integrated for required rehabilitation parameters in specific population by providing a VR game link to the patients' personal computers. Subsequently, therapists and rehabilitation professionals can prescribe the therapy protocols through the web based videoconferencing which are then accessed and executed by the patients through a given web browser. In this way, therapists can then monitor the patients' progress through the web and can also modify the therapy as required without real-time interaction or training. This program, apart from helping in adequate home care supervision also helps the professionals in maintaining connectivity with the caregivers or patients who have chronic illnesses and require long term follow up care. Decreasing the installation and running costs of such equipment's may expand their applicability in future. Moreover, especially for developing nations like ours, making it simpler and user-friendly so that it can be utilized by even computer illiterates and technologyunfriendly clients essential. Randomized trials correlating the clinical and functional recovery in terms of cognition and perception with changes in neuro-imaging especially functional imaging will further promote its utilization in clinical scenarios with confidence.

\section{References}

1. Weiss PL, Rand D, Katz N, Kizony R (2004) Video capture, virtual reality as a flexible and effective rehabilitation tool. J Neuroeng Rehabil 1: 12.

2. Aikat R, Dua V (2016) Mental imagery in spinal cord injury: A systematic review. J Spine 5: 310.

3. Pons JL, Torricelli D (2014) Emerging therapies in neuro-rehabilitation. Biosystems \& Biorobotics.

4. Brown ME, Gallimore JJ (1995) Visualization of three-dimensional structure during computer-aided design. Int $\mathrm{J}$ Human-Computer Interaction 7: 37-56.

5. Tyagi N, Nair P, Aikat R, Singh. JP. (2015) Case series illustrating the use of nintendo wiifit as an adjunct low cost tool for balance rehabilitation in Delhi/NCR . IJHSR. 5: 725-732.

6. Camara E, Rodriguez-Fornells A, Ye Z, Munte TF (2009) Reward networks in the brain as captured by connectivity measures. Front Neurosci 3: 350-362. 